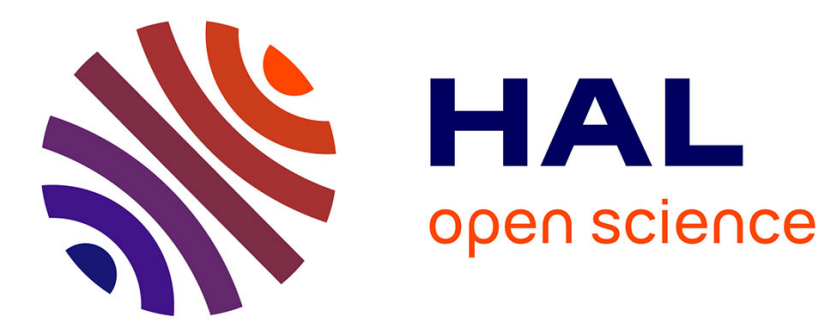

\title{
Transformation Methods for Static Field Problems With Random Domains
}

Duy Hung Mac, Stéphane Clenet, Jean-Claude Mipo

\section{To cite this version:}

Duy Hung Mac, Stéphane Clenet, Jean-Claude Mipo. Transformation Methods for Static Field Problems With Random Domains. IEEE Transactions on Magnetics, 2011, 47 (5), pp.1446-1449. 10.1109/TMAG.2010.2096460 . hal-00857179

\section{HAL Id: hal-00857179 https://hal.science/hal-00857179}

Submitted on 20 Sep 2013

HAL is a multi-disciplinary open access archive for the deposit and dissemination of scientific research documents, whether they are published or not. The documents may come from teaching and research institutions in France or abroad, or from public or private research centers.
L'archive ouverte pluridisciplinaire HAL, est destinée au dépôt et à la diffusion de documents scientifiques de niveau recherche, publiés ou non, émanant des établissements d'enseignement et de recherche français ou étrangers, des laboratoires publics ou privés. 


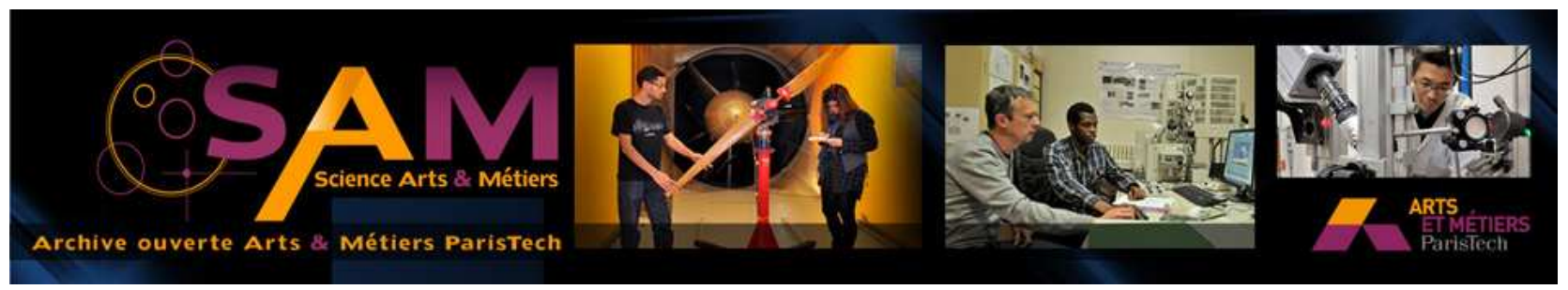

Science Arts \& Métiers (SAM)

is an open access repository that collects the work of Arts et Métiers ParisTech researchers and makes it freely available over the web where possible.

This is an author-deposited version published in: http://sam.ensam.eu

Handle ID: .http://hdl.handle.net/10985/7274

\section{To cite this version :}

Duy Hung MAC, Stéphane CLENET, Jean-Claude MIPO - Transformation Methods for Static Field Problems With Random Domains - IEEE Transactions on Magnetics - Vol. 47, n5, p.1446$1449-2011$ 


\title{
Transformation Methods for Static Field Problems with Random Domains
}

\author{
D.H. Mac ${ }^{1,2}$, S. Clénet ${ }^{1}$ and J.C. Mipo ${ }^{2}$ \\ ${ }^{1}$ L2EP/Arts et Métiers Paris Tech, 59000 Lille -France \\ ${ }^{2}$ VALEO-Systèmes Electriques, 94000 Créteil-France
}

\begin{abstract}
The numerical solution of Partial Differential Equations onto random domains can be done by using a mapping transforming this random domain into a deterministic domain. The issue is then to determine this one to one random mapping. In this paper, we present two methods - one based on the resolution of the Laplace equations, one based on a geometric transformation - to determine the random mapping. A stochastic magnetostatic example is treated to compare these methods.
\end{abstract}

Index Terms - Electromagnetic analysis, Stochastic Finite Element Method, Random Mapping, Random Domains

\section{INTRODUCTION}

The numerical resolution of the Maxwell equations enables the development of accurate models of electromagnetic systems. To solve numerically these partial differential equations, the Finite Elements Method (FEM) has been widely used. In several cases, the available input data are known with a finite level of confidence. These uncertainties can arise for instance from the aging of the materials or from imperfections of the manufacturing processes. Since the numerical models are more and more accurate due to the improvement of numerical methods (in 3D for example) and also due to the increase in computer performances, some of these uncertainties cannot be considered negligible any more. In several works, a probabilistic approach using random variables is used in order to take into account these uncertainties [1]. Methods have been presented in the literature to take into account the uncertainties on the behavior law [2], [3]. However, the case of uncertainties on the geometry is much less studied. In [4] one method which transforms the problem with uncertainties on the geometry into a problem with uncertainties on the behavior law is proposed. The challenge of this method is how to determine an efficient one to one random mapping that transforms the random domain into a deterministic domain.

In this paper, a comparison between two methods to calculate the random mapping is proposed. One is based on the resolution of the Laplace equations. The second is based on a geometric transformation. First, we present briefly the transformation method and we will show how the problem with uncertainties on the geometry can be transformed into a problem with uncertainties on the behavior law using a random mapping. Second, we will detail the two methods proposed to determine the random mapping addressed above. Finally, these two methods are compared on a stochastic magnetostatic example.

\section{TRANSFORMATION METHOD}

In this part, we will recall briefly the transformation method [5] used to solve electromagnetic problems with random domains.

The stochastic magnetostatic problem on a domain $\mathrm{D}(\boldsymbol{\theta})$ with random inner interfaces or boundaries can be written:

$$
\left\{\begin{array}{c}
\operatorname{div} \mathbf{B}(x, \boldsymbol{\theta})=0 \\
\operatorname{curl} \mathbf{H}(x, \boldsymbol{\theta})=0 \\
\mathbf{B}(x, \boldsymbol{\theta})=\boldsymbol{\mu}(x, \boldsymbol{\theta}) \mathbf{H}(x, \boldsymbol{\theta})
\end{array}\right.
$$

where $\boldsymbol{\theta}$, the outcome, refers to randomness of the problem. The uncertainties on the geometry can be taken into account by random interfaces $\Gamma_{k}(\boldsymbol{\theta})$ between two sub-domains $D_{i}$ and $D_{j}$. The permeability $\boldsymbol{\mu}$ depends on the position $x$ and also on the outcome $\boldsymbol{\theta}$. Actually, for a point $x$ located close to a random interface $\Gamma_{\mathrm{k}}(\boldsymbol{\theta})$, the value of the permeability depends on which side of $\Gamma_{\mathrm{k}}(\boldsymbol{\theta})$ the point $x$ belongs to. Therefore, the magnetic field $\mathbf{H}$ and the magnetic flux density $\mathbf{B}$ are also random fields.

We assume that the domain $\mathrm{D}(\boldsymbol{\theta})$ is bounded by the surface $\Gamma=\Gamma_{1} \cup \Gamma_{2} \cup \Gamma_{3}$ on which the boundary conditions are given by:

$$
\left\{\begin{array}{lr}
\mathbf{B}(x, \boldsymbol{\theta}) \cdot \mathbf{n}=0 & \text { on } \Gamma_{1}(\boldsymbol{\theta}) \\
\mathbf{H}(x, \boldsymbol{\theta}) \times \mathbf{n}=0 & \text { on } \Gamma_{2}(\boldsymbol{\theta}) \text { and } \Gamma_{3}(\boldsymbol{\theta})
\end{array}\right.
$$

where $\mathbf{n}$ is normal unit vector. The magnetomotive force $\gamma_{0}$ between $\Gamma_{2}(\boldsymbol{\theta})$ and $\Gamma_{3}(\boldsymbol{\theta})$ is imposed. This problem can be solved by using either the scalar or the vector potential formulation. In the following, the scalar potential formulation will be developed. If we denote $\Omega(x, \boldsymbol{\theta})$ the scalar potential that is a random field such that:

$$
\mathbf{H}(x, \boldsymbol{\theta})=-\operatorname{grad} \Omega(x, \boldsymbol{\theta})
$$

equation (1) can be written:

$$
\operatorname{div}(\mu(x, \theta) \operatorname{grad} \Omega(x, \theta))=0
$$

We obtain the weak formulation:

$$
\int_{D(\theta)}(\operatorname{grad} \Omega(x, \boldsymbol{\theta}))^{t} \boldsymbol{\mu}(x, \boldsymbol{\theta}) \operatorname{grad} \lambda(x, \boldsymbol{\theta}) \mathrm{d} x=0
$$

where $\lambda(x, \boldsymbol{\theta})$ is a scalar test function that is equal to zero on $\Gamma_{2}$ and $\Gamma_{3}$ and the superscript $t$ indicates the transpose of a matrix. In [5] we show that if there exists a one to one random mapping $X=X(x, \boldsymbol{\theta})$ which transforms the random domain $\mathrm{D}(\theta)$ to a reference domain $\mathrm{E}$ for each outcome $\boldsymbol{\theta}$, the weak formulation (5) written on $\mathrm{D}$ can be written on $\mathrm{E}$ :

$$
\int_{E}(\operatorname{grad} \Omega(X, \boldsymbol{\theta}))^{t} \boldsymbol{\mu}^{\prime}(X, \boldsymbol{\theta}) \operatorname{grad} \lambda(X) \mathrm{d} X=0
$$

where we have introduced the permeability tensor $\boldsymbol{\mu}^{\prime}(X, \boldsymbol{\theta})$ :

$$
\boldsymbol{\mu}^{\prime}(X, \boldsymbol{\theta})=\frac{\mathrm{M}^{t}(X, \boldsymbol{\theta}) \boldsymbol{\mu}(X, \boldsymbol{\theta}) \mathrm{M}(X, \boldsymbol{\theta})}{\mid \operatorname{det}(\mathrm{M}(X, \boldsymbol{\theta}) \mid}
$$


with $\mathrm{M}(\mathrm{X}, \boldsymbol{\theta})$ is the Jacobian matrix of the random mapping. The initial problem (1) with uncertain dimensions on the domain $\mathrm{D}$ is equivalent to a problem with uncertainties on a modified behavior law (permeability $\boldsymbol{\mu}^{\prime}(X, \boldsymbol{\theta})$ ) on the deterministic reference domain E. To solve this problem, one can use either intrusive methods (SSFEM) or non intrusive methods (NIM) [2], [3]. In this paper, a non intrusive method projection method [2] - has been used and will be briefly presented in the following.

We are interested in calculating an output random variable $\mathrm{W}(\boldsymbol{\theta})$ (energy stored in D for example). This random variable is approximated by:

$$
\mathrm{W}(\boldsymbol{\theta})=\sum_{i=1}^{N} w_{i} H_{i}(\boldsymbol{\theta})
$$

where $\left\{\mathrm{H}_{\mathrm{i}}(\boldsymbol{\theta})\right\}, \mathrm{i}=1, N$ a given orthogonal polynomial set (polynomial chaos) [6] and $\mathrm{w}_{\mathrm{i}}$ a real coefficient that is determined by the projection method:

$$
w_{i}=\frac{\mathrm{E}\left[\mathrm{W}(\boldsymbol{\theta}) H_{i}(\boldsymbol{\theta})\right]}{\mathrm{E}\left[H_{i}^{2}(\boldsymbol{\theta})\right]}
$$

where $\mathrm{E}[\mathrm{X}(\boldsymbol{\theta})]$ is the expectation of the random variable $\mathrm{X}(\boldsymbol{\theta})$. The calculation of the denominator can be done analytically whereas the calculation of the numerator can only be done numerically using a quadrature method. For the quadrature method, we consider several specific realizations (quadrature points $\boldsymbol{\theta}_{\mathrm{q}}$ ) of the random variable $\mathrm{W}(\boldsymbol{\theta})$ that corresponds to different deterministic geometries.

One can use the classical remeshing method (no mapping is required): for each quadrature point corresponding to a new geometry, the problem (5) is solved with a new mesh. This method is very time consuming. With the transformation method, we solve the problem (6) on the reference domain E. The permeability is revaluated using (7) for each quadrature point. The calculation is undertaken on a unique mesh of the reference domain $\mathrm{E}$, only the permeability distribution changes from a quadrature point to another. The difficulty is now how to determine the random mapping addressed above. In the following, we will discuss this aspect.

\section{RANDOM MAPPING DETERMINATION}

In the following, for simplicity, we will focus on the 2D case but an extension to 3D can be easily implemented. We consider a random domain $\mathrm{D}(\boldsymbol{\theta})$ which can be divided into $n$ subdomains $\mathrm{D}_{\mathrm{i}}(\boldsymbol{\theta})(\mathrm{i}=1, n)$ where the permeability is assumed to be constant. Each subdomain $\mathrm{D}_{\mathrm{i}}(\boldsymbol{\theta})$ is bounded by the random interface $\Gamma_{\mathrm{Di}}(\mathrm{c}, \boldsymbol{\theta})$ where $\mathrm{c}$ is the curvilinear coordinate. The random domain $\mathrm{D}(\boldsymbol{\theta})$ will be transformed by a one to one random mapping into a deterministic reference domain $\mathrm{E}$. This reference domain is also divided into $n$ subdomains $E_{i}$ bounded by $\Gamma_{\mathrm{Ei}}(\mathrm{c})$ (Fig. 1). This random mapping $\mathbf{K}(\boldsymbol{\theta})$ is completely defined when $n$ one to one random mappings $\mathbf{K}_{i}(\boldsymbol{\theta})$ transforming $\mathrm{D}_{\mathrm{i}}(\boldsymbol{\theta})$ into $\mathrm{E}_{\mathrm{i}}$ are determined. One constraint is that the common interface $\Gamma_{\mathrm{Dj}} \cap \Gamma_{\mathrm{Dk}}$ between two subdomains $\mathrm{D}_{\mathrm{j}}(\boldsymbol{\theta})$ and $\mathrm{D}_{\mathrm{k}}(\boldsymbol{\theta})$ must be transformed into a same surface by $\mathbf{K}_{j}(\boldsymbol{\theta})$ and by $\mathbf{K}_{k}(\boldsymbol{\theta})$. The one to one random mapping transforming $\Gamma_{\mathrm{Di}}(\mathrm{c}, \theta)$ into $\Gamma_{\mathrm{Ei}}(\mathrm{c})$ can be defined by following steps:

$$
\mathrm{P} \in \Gamma_{\mathrm{Di}}(\mathrm{c}, \boldsymbol{\theta}) \rightarrow \mathrm{c}_{\mathrm{P}} \rightarrow \mathrm{c}_{\mathrm{P}^{\prime}}=\mathrm{c}_{\mathrm{P}} \rightarrow \mathrm{P}^{\prime} \in \Gamma_{\mathrm{Ei}}(\mathrm{c})
$$

where $c_{P}$ and $c_{P}$, are the curvilinear coordinates of the points $P$ and $\mathrm{P}^{\prime}$ on the interface $\Gamma_{\mathrm{Di}}(\mathrm{c}, \boldsymbol{\theta})$ and $\Gamma_{\mathrm{Ei}}(\mathrm{c})$ respectively. The equality between the curvilinear coordinates $c_{p}$, and $c_{p}$ establishes a link between the boundaries of $E_{i}$ and $D_{i}(\theta)$ that is required to calculate the transformation $\mathbf{K}_{j}(\boldsymbol{\theta})$.

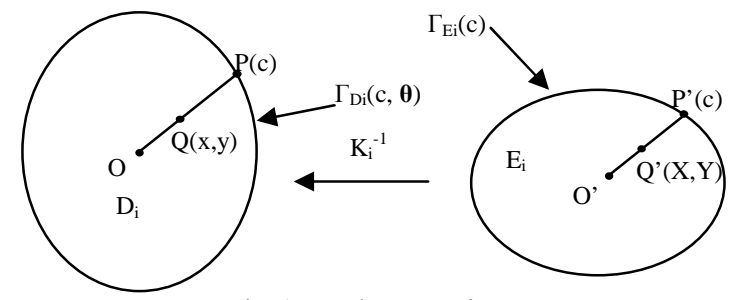

Fig. 1. Random mapping

We define $\mathbf{K}^{-1}{ }_{i}(\boldsymbol{\theta})$ the random mapping that transforms the domain $\mathrm{E}_{\mathrm{i}}$ into the random domain $\mathrm{D}_{\mathrm{i}}(\boldsymbol{\theta})$. In the following, since it is easier to determine the random mapping $\mathbf{K}^{-1}{ }_{i}(\boldsymbol{\theta})$ than $\mathbf{K}_{i}(\boldsymbol{\theta})$, we will detail the calculation of $\mathbf{K}^{\mathbf{- 1}}(\boldsymbol{\theta})$. The Jacobian matrix $\mathbf{M}_{i}(X, \boldsymbol{\theta})$ of $\mathbf{K}_{i}(\boldsymbol{\theta})$ is obtained easily by inverting the Jacobian matrix of $\mathbf{K}^{-\mathbf{1}}{ }_{i}(\boldsymbol{\theta})$. We detail now two methods to determine this random mapping $\mathbf{K}^{-\mathbf{1}}{ }_{i}(\boldsymbol{\theta})$.

\section{A. Laplace equations method}

This method was proposed by D. Xiu and D. Tartakovsky in [4]. The random mapping:

$$
\mathbf{K}_{i}^{-1}(\boldsymbol{\theta}):\left\{\begin{array}{l}
x=f(X, Y, \boldsymbol{\theta}) \\
y=g(X, Y, \boldsymbol{\theta})
\end{array}\right.
$$

is determined by solving the Laplace equations:

$$
\left\{\begin{array}{l}
\frac{\partial^{2} f(X, Y, \boldsymbol{\theta})}{\partial^{2} X}+\frac{\partial^{2} f(X, Y, \boldsymbol{\theta})}{\partial^{2} Y}=0 \\
\frac{\partial^{2} g(X, Y, \boldsymbol{\theta})}{\partial^{2} X}+\frac{\partial^{2} g(X, Y, \boldsymbol{\theta})}{\partial^{2} Y}=0
\end{array}\right.
$$

inside $\mathrm{E}_{\mathrm{i}}$ and satisfying the following boundary conditions:

$$
\left\{\begin{array}{l}
x_{P}(c, \boldsymbol{\theta})=f\left(X_{P^{\prime}}(c), Y_{P^{\prime}}(c), \boldsymbol{\theta}\right) \\
y_{P}(c, \boldsymbol{\theta})=g\left(X_{P^{\prime}}(c), Y_{P^{\prime}}(c), \boldsymbol{\theta}\right)
\end{array}\right.
$$

These boundary conditions enable the random mapping $\mathbf{K}^{-1}{ }_{i}(\boldsymbol{\theta})$ that transforms the boundary $\Gamma_{\mathrm{Ei}}(\mathrm{c})$ of $\mathrm{E}_{\mathrm{i}}$ into the boundary $\Gamma_{\mathrm{Di}}(\mathrm{c}, \boldsymbol{\theta})$ of $\mathrm{D}_{\mathrm{i}}(\boldsymbol{\theta})$ to be imposed (see (10)). In [4] stochastic differential equations (12) and (13) are rewritten as several deterministic differential equations by decomposing $f, g, x_{\mathrm{P}}$ and $y_{\mathrm{P}}$ under an expansion of $M$ mutually uncorrelated random variables $\mathrm{A}_{\mathrm{i}}(\theta)$, for example with $x_{p}$ and $f$ :

$$
\begin{aligned}
& x_{P}(c, \boldsymbol{\theta})=\sum_{j=1}^{M} x_{P j}(c) \cdot A_{j}(\boldsymbol{\theta}) \\
& f(x, y, \boldsymbol{\theta})=\sum_{j=1}^{M} f_{j}(x, y) \cdot A_{j}(\boldsymbol{\theta})
\end{aligned}
$$

Equations (12) and (13) yield $M$ deterministic equations:

$$
\left\{\begin{array}{l}
\frac{\partial^{2} f_{j}(X, Y)}{\partial^{2} X}+\frac{\partial^{2} f_{j}(X, Y)}{\partial^{2} Y}=0 \\
\frac{\partial^{2} g_{j}(X, Y)}{\partial^{2} X}+\frac{\partial^{2} g_{j}(X, Y)}{\partial^{2} Y}=0
\end{array}\right.
$$

inside $\mathrm{E}_{\mathrm{i}}$ with the following boundary conditions: 


$$
\left\{\begin{array}{l}
x_{P_{j}}(c)=f_{j}\left(X_{P^{\prime}}(c), Y_{P^{\prime}}(c)\right) \\
y_{P_{j}}(c)=g_{j}\left(X_{P^{\prime}}(c), Y_{P^{\prime}}(c)\right)
\end{array}\right.
$$

A collocation method based on an approximation of $f_{j}$ and $g_{j}$ by a Tchebychev polynomial expansion is proposed to solve these deterministic differential equations:

$$
\left\{\begin{array}{l}
f_{j}(X, Y)=\sum_{k=0}^{q_{1}} a_{k} \cdot T_{k}(X, Y) \\
g_{j}(X, Y)=\sum_{k=0}^{q_{2}} b_{k} \cdot T_{k}(X, Y)
\end{array}\right.
$$

where $T_{k}(X, Y)$ is a Tchebychev polynomial of order $k . a_{k}$ and $b_{k}$ are scalar coefficients that we have to determine.

The modeling of the boundary under the form (14) is not necessarily obvious. With the NIM method, it is not required to know $\mathbf{K}_{i}^{-1}(\boldsymbol{\theta})$ for all outcomes $\boldsymbol{\theta}$ but only for the $N$ quadrature points $\boldsymbol{\theta}_{\mathrm{q}}$ (see section II). Therefore we are interested only in the solution of (12) and (13) for $N$ given realizations $\boldsymbol{\theta}_{\mathrm{q}}$. Equation (12) and (13) now become deterministic equations. The collocation method based on an approximation of $f$ and $g$ by a Tchebychev polynomial expansion (17) could be directly applied here.

\section{B. Geometric transformation method}

We aim at finding for each point, Q', located inside the domain $E_{i}$ its transformation point, $Q$, in the domain $D_{i}$. For this task, we consider that the domain $\mathrm{E}_{\mathrm{i}}$ is composed of a set $\mathrm{S}\left(\mathrm{E}_{\mathrm{i}}\right)$ of uncountable segments (straight or curved). The intersection point- if it exists- of two segments is located only at one of their end points (the point $\mathrm{O}^{\prime}$ in the Fig. 1). Except this point, all other points Q' located inside domain $E_{i}$ belong to only one segment of $S\left(E_{i}\right)$. This constraint yields a one to one mapping $\mathbf{K}^{-1}(\boldsymbol{\theta})$. The domain $\mathrm{D}_{\mathrm{i}}$ is also divided in the same way. $\mathbf{K}_{i}^{-1}(\boldsymbol{\theta})$ is determined now by the one to one mapping defined onto $S\left(E_{i}\right)$ to $S\left(D_{i}\right)$. The domain $D_{i}(\theta)$ in Fig. 1 is divided by a set of segments $\mathrm{OP}(\mathrm{c}, \boldsymbol{\theta})$ and $\mathrm{E}_{\mathrm{i}}$ by a set of $\mathrm{O}^{\prime} \mathrm{P}^{\prime}(\mathrm{c})$ where $\mathrm{O}$ is a fixed point located inside $\mathrm{D}_{\mathrm{i}}$ and $\mathrm{O}^{\prime}$ inside $\mathrm{E}_{\mathrm{i}}$. The image OP under $\mathbf{K}^{-1}(\boldsymbol{\theta})$ of O'P' is obtained by giving the same curvilinear coordinates $\mathrm{c}$ for $\mathrm{P}$ and $\mathrm{P}^{\prime}($ see (10) ). Consider a point Q' inside $E_{\mathrm{i}}$. There exists one O'P' that $\mathrm{Q}^{\prime}$ belongs to. The image $\mathrm{Q}$ of $\mathrm{Q}^{\prime}$ under $\mathbf{K}^{-1}{ }_{i}(\theta)$ is located on OP and defined by:

$$
\overrightarrow{O Q}=\overrightarrow{O P} \cdot \frac{\left|O^{\prime} Q^{\prime}\right|}{\left|O^{\prime} P^{\prime}\right|}
$$

There are obviously several ways to divide a domain into a set of uncountable segments. The best choice is the one that gives the smoothest mapping and it depends on the actual geometry of the system studied. The use of a fixed point $\mathrm{O}$ and $\mathrm{O}$ ' is not mandatory as we will see in the application (see IV).

With this method, it seems difficult to obtain an analytical form of $\mathbf{K}^{-\mathbf{1}}{ }_{i}(\boldsymbol{\theta})$ and therefore, the Jacobian matrix can not be calculated explicitly. Each component of the Jacobian matrix at a point $\left(X_{0}, Y_{0}, \boldsymbol{\theta}_{\mathrm{q}}\right)$ is calculated numerically using a finite difference scheme where the mapping is calculated at $\left(X_{0}, Y_{0}, \boldsymbol{\theta}_{\mathrm{q}}\right)$ and $\left(X_{0}+\delta X_{0}, Y_{0}+\delta Y_{0}, \boldsymbol{\theta}_{\mathrm{q}}\right)$.

The main advantage of the transformation method compared to a method, based on the remeshing of each new geometry, is that it doesn't modify the connectivity between nodes. The connectivity modification adds additional variability on the output data. Moreover, additional data processing (mesh calculation) of the remeshing method is required which increases the time of calculation.

\section{NUMERICAL APPLICATION}

We consider now a magnetostatic problem defined in a random domain $D(\theta)$ presented in Fig. 2. The domain is divided in 4 areas $D_{i}, i=1,4$ with relative permeability $\mu_{1}=\mu_{2}=$ $\mu_{3}=1000$ and $\mu_{4}=1$. We impose a magnetomotive force $\gamma=$ 2A between $\Gamma_{1}$ and $\Gamma_{2}$ and $\mathbf{B} . \mathbf{n}=0$ on the remaining boundary [8], [9]. The uncertain dimensions (Fig. 2) are modeled by the uniform independent random variables $\mathrm{r}(\boldsymbol{\theta}), \mathrm{r}_{1}(\boldsymbol{\theta}), \mathrm{r}_{2}(\boldsymbol{\theta}), \mathrm{x}_{0}(\boldsymbol{\theta})$, $\mathrm{y}_{0}(\boldsymbol{\theta})$ where $\mathrm{r}$ and $\mathrm{x}_{0}, \mathrm{y}_{0}$ are the radius and the position of the disk $D_{3}, r_{1}$ and $r_{2}$ are the radius of the inside surface of the two teeth fronting of disk $\mathrm{D}_{3}$. The information of uniform independent random variables $r(\boldsymbol{\theta}), \mathbf{r}_{1}(\boldsymbol{\theta}), \mathrm{r}_{2}(\boldsymbol{\theta}), \mathbf{x}_{0}(\boldsymbol{\theta}), \mathrm{y}_{0}(\boldsymbol{\theta})$ is given by the Table I. The aim is to calculate the energy $\mathrm{W}(\boldsymbol{\theta})$ stored in the domain $\mathrm{D}(\boldsymbol{\theta})$.
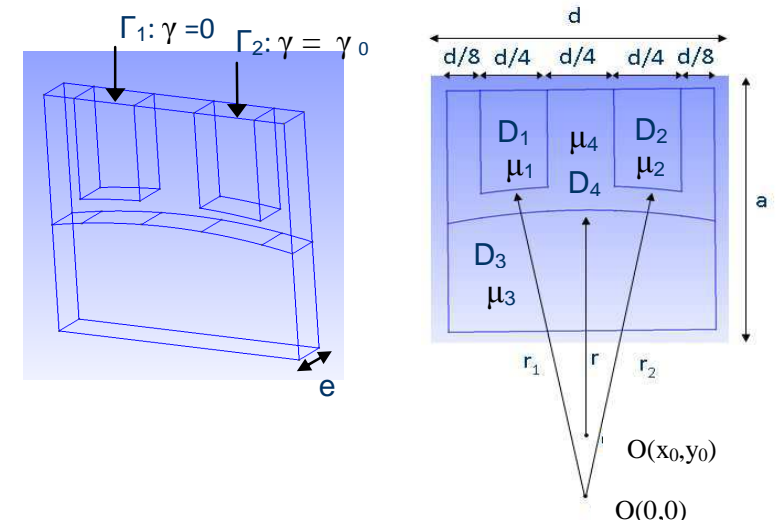

Fig. 2. Magnetostatic system

In the following, we will detail the determination of the mapping for one quadrature point $\boldsymbol{\theta}_{\mathrm{q}}$ (section II) for each method A (section III.A) and B (section III.B). We take the reference domain $E$ with the following dimensions: $r_{1}=R_{1}$, $r_{2}=R_{2}, r=R, x_{0}=X_{0}, y_{0}=Y_{0}$ which are the mean values of these random variables (see Table I). The first step consists in dividing efficiently the reference domain $\mathrm{E}$ into several subdomains $\mathrm{E}_{\mathrm{i}}$. The domain decomposition for this problem is presented in Fig. 3.
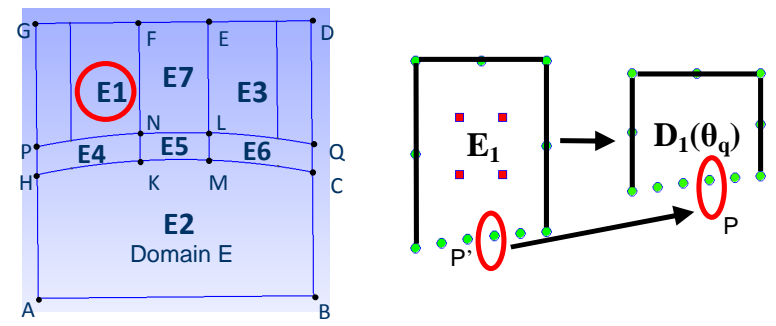

Fig. 3. Division of domain E and the mapping defined by the method A

We focus on a random mapping that transforms the subdomain $E_{1}$ corresponding to $r_{1}=R_{1}$ (domain bounded by $N$ F-G-P in Fig. 3) into subdomain $D_{1}$ corresponding to the outcome $\boldsymbol{\theta}_{\mathrm{q}}$ with $\mathrm{r}_{1}=\mathrm{R}_{1}$, 
For the method A, the mapping is obtained by solving equations (12) and (13) with the outcome $\boldsymbol{\theta}_{\mathrm{q}}$ corresponding to $\mathrm{r}_{1}=\mathrm{R}_{1}$. We take $\mathrm{q}_{1}=\mathrm{q}_{2}=14$ for (17). To calculate the coefficients $a_{k}$ and $b_{k}$ in (17), we have to choose 15 collocation points to obtain a non singular linear equation system (Fig. 3). The interior points (square points) satisfy (12) and the boundary points (round points) satisfy (13) providing that the relationship between $\mathrm{P}$ located on the boundary of $\mathrm{D}_{1}$ and $\mathrm{P}$ ' located on the boundary of $E_{1}$ is determined by (10).

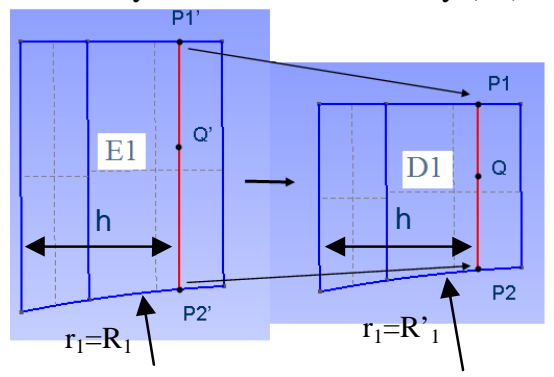

Fig. 4. The mapping based on the method B

For the method $\mathrm{B}$, domain $\mathrm{E}_{1}$ and $\mathrm{D}_{1}$ can be considered as a set of straight parallel segments $\mathrm{P}_{1}{ }^{\prime} \mathrm{P}_{2}$ ' and $\mathrm{P}_{1} \mathrm{P}_{2}$ respectively. The curvilinear coordinate used here is the distance $\mathrm{h}$ with the left vertical side GP of $\mathrm{E}_{1}$ (Fig.1 and Fig. 4). The transformation Q' into $\mathrm{Q}$ is defined by the following relationship:

$$
\frac{\overrightarrow{Q P_{1}}}{\overrightarrow{Q P_{2}}}=\frac{\overrightarrow{Q^{\prime} P_{1}^{\prime}}}{\overrightarrow{Q^{\prime} P_{2}^{\prime}}}
$$

TABLE I

INPUT DATA INFOMATIONS

\begin{tabular}{|c|c|c|c|c|c|}
\hline & $\mathrm{r}(\mathrm{cm})$ & $\mathrm{r}_{1}(\mathrm{~cm})$ & $\mathrm{r}_{2}(\mathrm{~cm})$ & $\mathrm{x}_{0}(\mathrm{~cm})$ & $\mathrm{y}_{0}(\mathrm{~cm})$ \\
\hline Lower bound & 49.97 & 50.27 & 50.27 & -0.003 & -0.003 \\
\hline Upper bound & 50.03 & 50.33 & 50.33 & 0.003 & 0.003 \\
\hline Mean value & 50 & 50.3 & 50.3 & 0 & 0 \\
\hline
\end{tabular}

In Table II, we present the mean value and the standard deviation of the energy $\mathrm{W}(\theta)$ stored in the domain $\mathrm{D}(\theta)$ obtained by two method A and B using the scalar potential and the vector potential formulations [7]. A mesh of 4700 nodes has been used. In this magnetostatic problem the numerical error can be evaluated by the gap between the mean value of energy obtained by scalar potential and vector potential formulations [7]. We can notice that the errors obtained by two methods are relatively small and almost the same. This means that the choice of either method A or method B has few influences on the result. The main advantage of random mapping determined by method $\mathrm{A}$ is that it can be programmed systematically. However, this method has some drawbacks. It requires a numerical resolution of (12) and (13) where some numerical error can be generated. Therefore, the random mapping does not transform exactly $E_{i}$ into $D_{i}$. Moreover, a common interface between two subdomains $D_{j}(\boldsymbol{\theta})$ and $\mathrm{D}_{\mathrm{k}}(\boldsymbol{\theta})$ can not be exactly transformed into a same surface by $\mathbf{K}_{j}(\boldsymbol{\theta})$ and by $\mathbf{K}_{k}(\boldsymbol{\theta})$. Furthermore, with non convex domains, it is possible that this mapping transforms the point $\mathrm{P}$ ' located inside $E_{i}$ into a point $P$ outside $D_{i}$. This yields significant numerical error on the output. The geometric transformation (method B) can avoid the drawbacks of method
A. It should be mentioned that for domains with complex geometries, it is possible to divide the domain into elementary subdomains (CAD tools naturally provide this decomposition) on which the method B can be applied to each of them. Finally, in our example with a mesh of 4700 nodes, the method $\mathrm{B}$ is faster than the method $\mathrm{A}$ with a time calculation ratio of about 2 . However, we should mention that in the method B, an infinity of geometric transformations give the same solution in the continuous domain but not in the discrete domain. In fact, it can be shown that the numerical error strongly depends on the choice of the geometric transformation. Criteria should be defined in order to determine the transformation that introduces the smallest numerical error.

TABLE II

METHOD A COMPARED WITH METHOD B

\begin{tabular}{|c|c|c|c|c|}
\hline \multirow{2}{*}{ Information } & \multicolumn{2}{|c|}{ Method A } & \multicolumn{2}{c|}{ Method B } \\
\cline { 2 - 5 } & $\begin{array}{c}\text { Scalar } \\
\text { formulation }\end{array}$ & $\begin{array}{c}\text { Vector } \\
\text { formulation }\end{array}$ & $\begin{array}{c}\text { Scalar } \\
\text { formulation }\end{array}$ & $\begin{array}{c}\text { Vector } \\
\text { formulation }\end{array}$ \\
\hline Mean value & 138.98 & 134.31 & 138.97 & 134.31 \\
\hline $\begin{array}{c}\text { Standard } \\
\text { deviation }\end{array}$ & 9.25 & 8.83 & 9.22 & 8.82 \\
\hline
\end{tabular}

\section{CONCLUSION}

We have presented and compared two methods to determine the random mapping used to solve the problem with random domain. The example presented above shows that the results obtained by the two methods are almost the same. However the method $\mathrm{B}$ is simpler to implement and less time consuming.

\section{REFERENCES}

[1] R. Ghanem, P. D. Spanos, Stochastic Finite Elements: A spectral approach. Mineola, NY: Dover 2003

[2] R. Gaignaire, S. Clenet, O. Moreau, and B. Sudret. 3D spectral stochastic finite element method in electromagnetism. IEEE Trans.Magn. vol.43, no.4, pp. 1209-1212, 2007.

[3] M. Berveiller, B. Sudret and M. Lemaire, Stochastic finite element: a non intrusive approach by regression, Rev. Eur. Méca. Vol. 15, N¹-23/2006, pp. 81-92. (2006)

[4] D. Xiu and D. M. Tartakovsky. Numerical methods for differential equations in random domains. SIAM J.SCI COMPUT. No 3, pp.1167$1185,2006$.

[5] D.H. Mac, S. Clenet, J.C. Mipo, O.Moreau. Solution for static field problem in random domains. IEEE Trans.Magn, vol.46, no.8, pp. 33853388, 2010.

[6] D. Xiu and G. Karniadakis, The Wiener Askey polynomial chaos for static differential equations, SIAM J. Sci. Comput. Vol.24, No.2, pp. 619-644. 2002

[7] S. Clenet and N. Ida, Error estimation in a stochastic finite element method in electrokinetics, Int. J. Numer. Meth. Engng 2010; 81:14171438.

[8] T. Henneron, Y. Le Menach, F. Piriou, O. Moreau, S. Clenet, J.P Ducreux and J.C. Verite. Source field computation in NDT applications. IEEE Trans.Magn. vol.43, issue 4, pp. 1785-1788, 2007.

[9] P. Dular, W. Legros and A. Nicolet. Coupling of local and global quantities in various finite element formulations and its application to electrostatics, magnetostatics and magnetodynamics. IEEE Trans.Magn. vol.34, issue 5, pp. 3078-3081, 1998.

\section{ACKNOWLEDGEMENT}

This work is supported by the program MEDEE funded by the Nord Pas de Calais council and the European Community. 